\title{
Aportaciones de Paulo Freire a la educación intercultural. Un estudio de caso en la Educación Secundaria Obligatoria
}

\author{
María Verdeja Muñiz; Xosé Antón González Riaño*
}

Resumen. La finalidad general de nuestra investigación es sintetizar las aportaciones de la pedagogía de Paulo Freire a la educación intercultural y estudiar las posibilidades de aplicación al sistema educativo. La investigación está planteada desde una perspectiva cualitativa y completada con un estudio de caso. En un primer momento realizamos un análisis de una parte seleccionada de la obra escrita de Freire y establecemos las líneas básicas de lo que sería su sensibilidad intercultural. En otro plano de análisis realizamos un estudio de las materias del currículo de la ESO en Asturias en su dimensión intercultural. Las aportaciones de la pedagogía de Paulo Freire nos han permitido identificar retos que la diversidad cultural plantea al currículo asturiano. El estudio de caso en el centro educativo pone de manifiesto que, para llevar a cabo verdaderas propuestas de educación intercultural, sería necesario que existieran unas condiciones previas que tienen que ver, entre otros, con los siguientes aspectos: la existencia de una infraestructura organizativa y conceptual en el centro en la que toda la vida académica se "interculturalice", la necesidad de asumir con convicción el sentido educativo y social de la enseñanza secundaria y desarrollar un modelo de enseñanza comprensiva, apostar por un modelo de currículo en el que tenga cabida la diversidad cultural del alumnado. Todo ello, además, en un contexto en el que el alumnado tenga igualdad de oportunidades para desarrollarse desde un punto de vista social, cultural y educativo.

Palabras clave: educación intercultural; diversidad lingüística; diálogo; Paulo Freire; política.

CONTRIBUIÇÕES DE PAULO FREIRE À EDUCAÇÃO INTERCULTURAL. UM ESTUDO DE CASO NO ENSINO SECUNDÁRIO OBRIGATÓRIO

Resumo. O objetivo geral de nossa pesquisa é sintetizar as contribuições da pedagogia de Paulo Freire para a educação intercultural e estudar as possibilidades de aplicação no sistema de ensino. A pesquisa está estruturada a partir de uma perspectiva qualitativa e também apresenta um estudo de caso. Inicialmente, fizemos uma análise de uma parte selecionada do trabalho escrito de Freire e estabelecemos as linhas básicas do que seria sua sensibilidade intercultural. Em outra análise, realizamos um estudo das matérias do currículo da ESO em Astúrias (Espanha) em sua dimensão intercultural. As contribuições da pedagogia de Paulo Freire

* Departamento de Ciencias de la Educación, Facultad de Formación del Profesorado y Educación, Universidad de Oviedo, España. 
nos permitiram identificar os desafios que a diversidade cultural coloca para o currículo asturiano. O estudo de caso na escola mostra que, para realizar propostas concretas de educação intercultural, seria necessário que existissem algumas condições prévias relacionadas, entre outros, aos seguintes aspectos: a existência de uma infraestrutura organizacional e conceitual na escola, onde toda a vida acadêmica se "interculturalize"; a necessidade de assumir com convicção o sentido educacional e social do ensino secundário; o desenvolvimento de um modelo de ensino abrangente e um modelo de currículo com espaço para a diversidade cultural dos alunos. Tudo isso, além disso, em um contexto em que os alunos tenham as mesmas oportunidades de se desenvolverem do ponto de vista social, cultural e educacional.

Palavras-chave: educação intercultural; diversidade linguística; diálogo; Paulo Freire; política.

CONTRIBUTIONS OF PAULO FREIRE'S TO INTERCULTURAL EDUCATION. A CASE STUDY IN SECONDARY EDUCATION

Abstract. The general purpose of the research is to investigate and reflect on the contributions of Paulo Freire's pedagogy to intercultural education and study its applicability in the educative system. The research that we have developed is of qualitative character and is focused on a case study. In a first level, we make an analysis of a selected portion of the written work of Freire and we establish the basic lines of what would be their intercultural sensitivity. On another level of analysis we conducted a study in the curriculum of the ESO in Asturias in its intercultural dimension. The contributions Paulo Freire have allowed us to identify challenges that the cultural diversity present in the Asturias curriculum. The results obtained in our study make it clear that, despite the appropriateness of the present moment, the need for the development of this proposal and the fact that the educational community is willing to incorporate; it would be necessary that determined prior conditions should exist. These conditions have to do fundamentally with aspects such as: organizational infrastructure in the school where all academic life it becomes intercultural; understand the educational and social meaning of secondary education, the need to launch a curriculum in which, cultural diversity has its own place, and all of this, within a context where the students enjoy the same educational opportunities in order to grow from a social, cultural and educational.

Keywords: intercultural education; linguistic diversity; dialogue; Paulo Freire; politics. 


\section{INTRODUCCIÓN}

En el contexto social y educativo actual el fenómeno de la multiculturalidad se destaca como una característica básica de nuestra sociedad. Dicho fenómeno es bien visible en los centros y contextos educativos de la comunidad autónoma asturiana (Louzao y González Riaño, 2007). La preocupación por la multiculturalidad en el contexto educativo ha Ilevado a que diversos autores planteen los retos educativos que conlleva educar en sociedades multiculturales (Lynch, 1991; Merino y Muñoz Sedano, 1995; Sáez Alonso, 2006; Torres Santomé, 2008; Pérez Tapias, 2010; Banks, 2015) desarrollándose diversos estudios e investigaciones (Sleeter y Grant, 1999; Louzao, 2009; Aguado, 2011; Díez Gutiérrez, 2014) y convirtiéndose en un campo de investigación con identidad propia.

Por otro lado, diferentes autores (Durkheim, 1975; Pérez Gómez, 1994; García Castaño, Pulido y Montes 1997; Gimeno, 2005), procedentes de diferentes disciplinas, vienen haciendo referencia a la función socializadora de la escuela y a su papel en la transmisión de la cultura. A pesar de la existencia de otros agentes socializadores -familia, sociedad, medios de comunicación-, parece ser que hay acuerdo en que la escuela representa uno de los principales agentes socializadores y que desempeña un importante papel en la transmisión de la cultura y conocimientos a las nuevas generaciones. Ahora bien, cabe preguntarse: ¿qué modelo cultural debe transmitir la escuela a las nuevas generaciones? o, lo que es más importante: ¿está la escuela preparada para la multiculturalidad? Responder a estas cuestiones no es tarea sencilla y en ese sentido podemos decir que la educación en contextos multiculturales continúa siendo un debate abierto.

Cuando planteamos este trabajo de investigación asumimos que el gran educador brasileño Paulo Freire (1921-1997) debe ser considerado un referente fundamental de la educación intercultural. Su pedagogía, en efecto y como señala Besalú (2002), parte del principio de que para educar a las personas hay que conocerlas, respetarlas y acogerlas en su diversidad cultural.

Es preciso señalar -para que no exista confusión-que cuando hablamos de diversidad cultural y educación en contextos educativos multiculturales, nos referimos a una educación dirigida a todo el alumnado en general (no solo al alumnado extranjero). Esto mismo también es aplicable cuando hablamos de educación intercultural. Simplemente queremos dejar constancia que los contextos educativos actuales son multiculturales - diversidad de culturas-y una escuela multicultural es una escuela en la que conviven estudiantes con diferentes identidades culturales ya sean nacionales o extranjeros. Teniendo en cuenta esto, cuando hablamos de educación intercultural, pensamos en una educación dirigida a todo el alumnado en general. 


\section{OBJETIVOS DEL ESTUDIO}

La finalidad general de la investigación que presentamos es indagar y reflexionar acerca de las aportaciones de la pedagogía de Paulo Freire a la educación intercultural y estudiar las posibilidades de aplicación en el sistema educativo asturiano. A partir de esta finalidad genérica, planteamos los siguientes objetivos:

- Revisar, analizar y sintetizar, a partir de obras seleccionadas de Paulo Freire su visión acerca de la educación intercultural.

- Conocer los principales retos que la diversidad cultural incorpora al currículo de ESO en Asturias y establecer, en su caso, relaciones con aportaciones de Freire.

- Formular una alternativa inicial de educación intercultural para la ESO, de acuerdo con los presupuestos teóricos anteriores.

- Contrastar la posibilidad de aplicación de tal alternativa a partir de un estudio de caso, en un IES asturiano.

- Establecer unos principios coherentes de educación intercultural de aplicación más genérica al marco educativo asturiano.

La visión sintética de nuestra investigación se podría reflejar en la figura 1 , en la que ponemos en relación las estructuras macro-contexto y micro-contexto del estudio.

\section{MÉTODO}

Desde el punto de vista metodológico optamos por un enfoque de investigación de carácter cualitativo, completado con un estudio de caso de orientación etnográfica. El estudio se realizó durante el curso 2014-15 en un Instituto de Enseñanza Secundaria de Oviedo. Se trata de un centro con más de 150 años de historia, con gran tradición en Asturias y al que actualmente acude gran cantidad de alumnado extranjero. Plantear la investigación con una metodología cualitativa responde a la finalidad de comprender a fondo la realidad y el contexto en el que nos encontramos, poniendo especial interés en comprender los puntos de vista de sus protagonistas. Al mismo tiempo y en coherencia con San Fabián (2011) la investigación sobre las instituciones educativas no puede entenderse al margen de las teorías de las organizaciones $y$, en ese sentido, nos decantamos por esta metodología porque nos brinda la posibilidad de plantear un caso que contemple un análisis en profundidad y multidimensional teniendo en cuenta, lógicamente, el modelo organizacional. 


\section{FIGURA 1}

\section{Relaciones entre estructuras macro-contexto y micro-contexto}

\section{RELACIONES ENTRE LAS ESTRUCTURAS MACRO Y MICRO CONTEXTO}

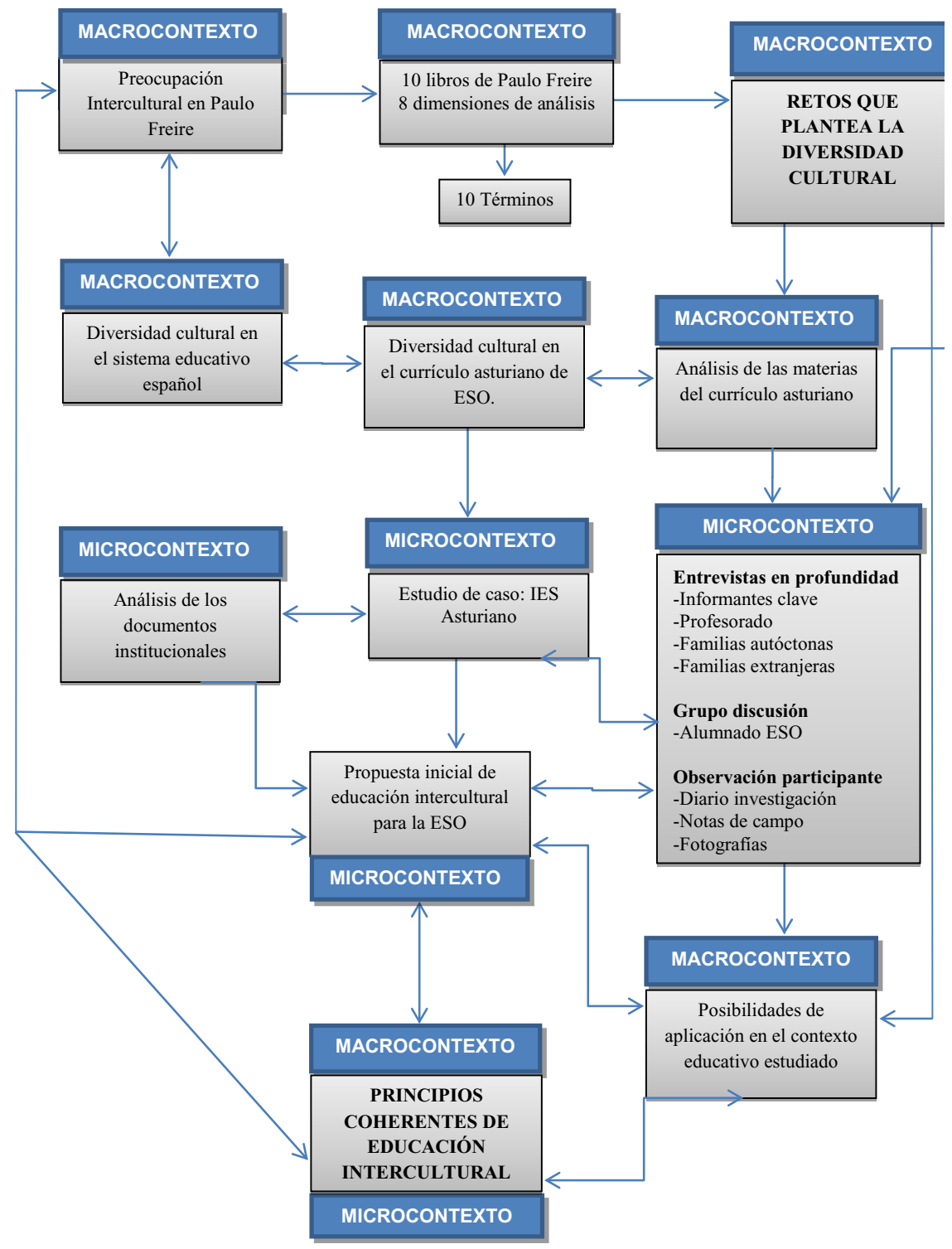




\section{MUESTRA}

Con relación a la selección y características de la muestra conviene recordar que en etnografía es habitual utilizar el muestreo teórico (Goetz y LeCompte, 1988) así pues, éste ha sido el procedimiento de muestro que utilizamos en nuestra investigación. Para ello pusimos especial esmero en escoger a los informantes. La muestra la componen los siguientes grupos de informantes: profesorado experto (5) otro profesorado de secundaria (10). Para escoger a este grupo de informantes hemos tenido en cuenta dos criterios: 1) infamantes que aportasen diversidad de puntos de vista, 2) informantes "expertos" o familiarizados con el tema objeto de estudio, lo que nos brindaría una visión holística de la temática. Otro grupo de informantes son las familias -autóctonas y extranjeras- agrupadas de la siguiente manera: autóctonas (5) y familias extranjeras (5). En el caso de las familias nuestro criterio principal guarda relación con la diversidad de familias. Con relación al alumnado la muestra la forman 74 sujetos. Para escoger a este grupo de informantes tuvimos en cuenta dos criterios: 1) grupos de alumnado en los que exista diversidad cultural y 2) grupos pertenecientes a diferentes cursos de secundaria.

\section{TÉCNICAS DE RECOGIDA Y ANÁLISIS DE LA INFORMACIÓN}

Las técnicas de recogida de información se plantearon en coherencia con el modelo de investigación cualitativa. Hemos tenido en cuenta aquellas que diversos autores -Goetz y LeCompte, 1988; González Riaño, 1994-, consideran inequívocamente etnográficas. En la tabla 1 presentamos las técnicas de recogida y análisis de la información que utilizamos:

\section{TABLA 1}

Técnicas de recogida y análisis de la información

\begin{tabular}{|l|l|l|}
\hline $\begin{array}{c}\text { Técnica de } \\
\text { recogida de } \\
\text { información }\end{array}$ & \multicolumn{1}{|c|}{$\begin{array}{c}\text { Fuente de recogida de } \\
\text { datos }\end{array}$} & $\begin{array}{l}\text { Técnica utilizada para el análisis de } \\
\text { la información }\end{array}$ \\
\hline \multirow{2}{*}{$\begin{array}{l}\text { Análisis documen- } \\
\text { tal }\end{array}$} & $\begin{array}{l}\text { Libros en castellano de Freire } \\
(10)\end{array}$ & $\begin{array}{l}\text { Ocho dimensiones de análisis y } 10 \text { términos } \\
\text { (relacionados educación intercultural). }\end{array}$ \\
\cline { 2 - 3 } & Currículo asturiano (ESO) & $\begin{array}{l}\text { Cinco dimensiones de análisis (relaciona- } \\
\text { das con la educación intercultural). }\end{array}$ \\
\cline { 2 - 3 } & Documentos institucionales & Cuestionario de evaluación inicial \\
\hline $\begin{array}{l}\text { Entrevistas en pro- } \\
\text { fundidad } \\
\text { (25 sujetos) }\end{array}$ & $\begin{array}{l}\text { Profesorado experto (5) } \\
\text { Profesorado IES (10) } \\
\text { Familias autóctonas (5) } \\
\text { Familias extranjeras (5) }\end{array}$ & Análisis a partir de categorías emergentes \\
\hline
\end{tabular}




\begin{tabular}{|l|l|l|}
\hline $\begin{array}{c}\text { Técnica de } \\
\text { recogida de } \\
\text { información }\end{array}$ & \multicolumn{1}{|c|}{$\begin{array}{c}\text { Fuente de recogida de } \\
\text { datos }\end{array}$} & $\begin{array}{l}\text { Técnica utilizada para el análisis de } \\
\text { la información }\end{array}$ \\
\hline $\begin{array}{l}\text { Observación Parti- } \\
\text { cipante }\end{array}$ & $\begin{array}{l}\text { Notas de campo } \\
\text { Diario de investigación } \\
\text { Fotografías }\end{array}$ & $\begin{array}{l}\text { Criterios: 1) La especificación de contextos. } \\
\text { 2) Descripción del ambiente y espacios. 3) } \\
\text { Identificación de actividades de participan- } \\
\text { tes. 4) Las interacciones informales. 5) El } \\
\text { lenguaje de los participantes. }\end{array}$ \\
\hline $\begin{array}{l}\text { Grupos de Discu- } \\
\text { sión (74 sujetos) }\end{array}$ & Alumnado ESO (5 grupos) & $\begin{array}{l}\text { Análisis a partir de categorías previas y } \\
\text { emergentes }\end{array}$ \\
\hline
\end{tabular}

\section{PROCEDIMIENTO Y ANÁLISIS DE LA INFORMACIÓN DEL ANÁLISIS DOCUMENTAL}

\subsection{Análisis documental de la bibliografía seleccionada de Paulo Freire}

Es importante señalar que entendemos que los libros de Freire utilizados para el análisis constituyen una serie de documentos personales donde el autor reflexiona acerca de diversas temáticas. Entendemos que las aportaciones bibliográficas del educador brasileño pueden ser tratadas como auténticos documentos personales y pueden ser utilizados para efectuar un análisis documental de carácter cualitativo. Hemos escogido diez libros que, consideramos, constituyen una visión representativa de su extensa obra. La elección de tales fuentes guarda relación con dos criterios: 1) libros escritos en castellano, y 2) libros de todas las etapas de su vida. En la tabla 2, mostramos los libros, dimensiones y términos utilizados para realizar nuestro análisis:

\section{TABLA 2}

\section{Libros de Freire, dimensiones y términos utilizados en el análisis}

\begin{tabular}{|c|c|c|}
\hline $\begin{array}{c}\text { Bibliografía seleccionada de Paulo } \\
\text { Freire }\end{array}$ & $\begin{array}{c}\text { Dimensiones de } \\
\text { análisis }\end{array}$ & Términos \\
\hline $\begin{array}{l}\text { 1) Educación como práctica de la libertad } \\
\text { (1969) } \\
\text { 2) Pedagogía del oprimido (1970) } \\
\text { 3) Pedagogía de la esperanza: un reencuentro } \\
\text { con la Pedagogía del oprimido (1993) } \\
\text { 4) La naturaleza política de la educación (1994) } \\
\text { 5) Pedagogía de la autonomía. Saberes nece- } \\
\text { sarios para la práctica educativa (1997) } \\
\text { 6) A la sombra de este árbol (1997a) } \\
\text { 7) El grito manso (2003) } \\
\text { 8) Pedagogía de la tolerancia (2006) } \\
\text { 9) Pedagogía de la indignación (2010) } \\
\text { 10) Cartas a quien pretende enseñar (2012) }\end{array}$ & $\begin{array}{l}\text { 1) Diversidad Cultural } \\
\text { 2) Concepción antropo- } \\
\text { lógica de cultura } \\
\text { 3) Cultura dominante } \\
\text { 4) El diálogo como méto- } \\
\text { do de conocimiento } \\
\text { 5) Concepto de Edu- } \\
\text { cación } \\
\text { 6) Virtudes inherentes a } \\
\text { la práctica docente } \\
\text { 7) Crítica de Freire a la } \\
\text { educación bancaria } \\
\text { 8) Escuela democrática }\end{array}$ & $\begin{array}{l}\text { 1) Tolerancia } \\
\text { 2) Diversidad } \\
\text { 3) Identidad Cul- } \\
\text { tural } \\
\text { 4) Diálogo } \\
\text { 5) "El Otro" } \\
\text { 6) Escuela Demo- } \\
\text { crática } \\
\text { 7) Convivencia } \\
\text { 8) Multiculturalidad } \\
\text { 9) Política } \\
\text { 10) Transformación }\end{array}$ \\
\hline
\end{tabular}


Para realizar el análisis de la información obtenida a partir de la bibliografía seleccionada de Freire, primeramente, hemos planteado ocho dimensiones de análisis relacionadas con los objetivos del estudio. Para continuar con el análisis cualitativo fuimos escogiendo citas textuales del autor brasileño en las que aparecían términos que previamente habíamos seleccionado para efectuar dicho análisis. El análisis cualitativo efectuado nos ha permitido constatar la sensibilidad intercultural del autor y nos ha brindado la oportunidad de guiar nuestra mirada hacia cuestiones de suma importancia en el contexto de nuestra investigación ya que hemos podido identificar ideas centrales de su pensamiento así como algunas de sus preocupaciones, identificando, además, temáticas recurrentes en el autor.

\subsection{Análisis de las materias del currículo asturiano de la etapa de secundaria}

Para identificar posibles retos que la diversidad cultual plantea al currículo asturiano en la etapa de la Educación Secundaria Obligatoria, realizamos una revisión de las materias que configuran el currículo. En el momento de realización del estudio estaba vigente el Decreto 74/2007 de 14 de junio por el que se ordena y establece el currículo en la etapa de la Educación Secundaria Obligatoria. Dicha revisión la efectuamos a partir las dimensiones de análisis que tiene que ver con diferentes elementos del currículo, como así representamos en la figura 2:

\section{FIGURA 2}

\section{Dimensiones de análisis del currículo asturiano}

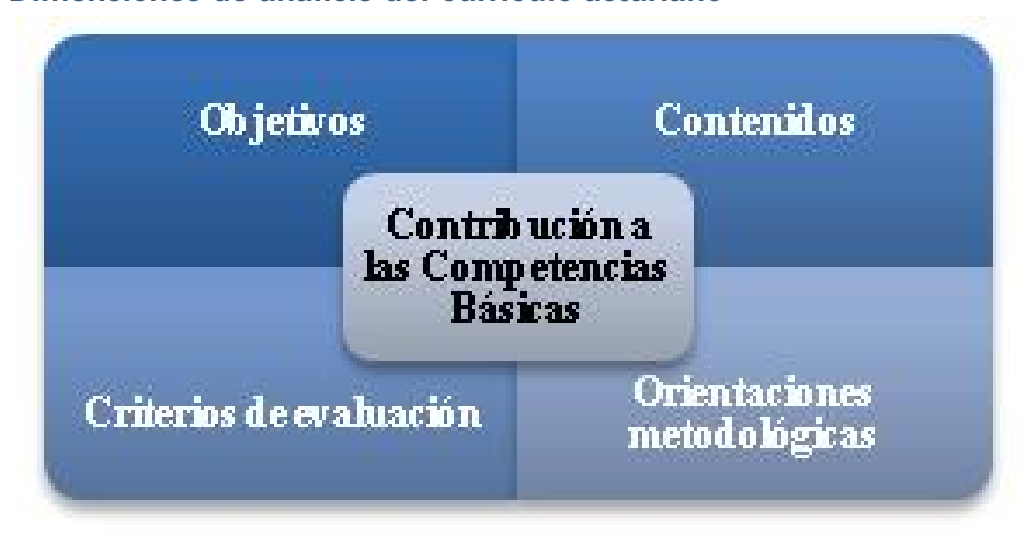


Para efectuar el análisis del currículo asturiano hemos consultado cada una de las materias del mismo y, tomando como referencia los siguientes elementos: objetivos, contenidos, orientaciones metodológicas, criterios de evaluación y contribución a las competencias básicas, efectuamos un análisis cualitativo poniendo nuestra mirada en la dimensión intercultural.

\subsection{Análisis documental de los documentos institucionales}

Para realizar el análisis de documentos institucionales nos hemos apoyado inicialmente en un instrumento ya elaborado en el contexto de Proyecto Atlántida (García, 2003), utilizado para la evaluación del tratamiento de los procesos de interculturalidad en los centros educativos. Así pues, tomando como referencia tal instrumento, lo hemos adaptado a la realidad del contexto de nuestro estudio. Una primara revisión de documentos institucionales se haría en torno a preguntas planteadas en este cuestionario:

\section{TABLA 3}

\section{Cuestionario utilizado para el análisis de documentos institucionales}

\begin{tabular}{|c|c|c|}
\hline Preguntas para el análisis & si & no \\
\hline $\begin{array}{l}\text { Existen objetivos relacionados con la diversidad cultural en el Proyecto Educativo } \\
\text { de Centro. }\end{array}$ & & \\
\hline $\begin{array}{l}\text { Se ha realizado una revisión y evaluación de las actitudes del profesorado respecto } \\
\text { a la educación intercultural. }\end{array}$ & & \\
\hline $\begin{array}{l}\text { Existe un Plan de Acogida con actuaciones al inicio del curso para recibir al alumnado } \\
\text { que se incorpora de forma tardía. }\end{array}$ & & \\
\hline $\begin{array}{l}\text { En los documentos institucionales se entiende la interculturalidad como un factor } \\
\text { enriquecedor y positivo del centro. }\end{array}$ & & \\
\hline $\begin{array}{l}\text { Están determinadas las actuaciones a realizar por los tutores/as con alumnos/as } \\
\text { extranjeros. }\end{array}$ & & \\
\hline El ambiente escolar del centro está abierto al hecho multicultural. & & \\
\hline $\begin{array}{l}\text { Se asume la defensa del pluralismo cultural y el compromiso contra la discriminación } \\
\text { como rasgo de identidad. }\end{array}$ & & \\
\hline $\begin{array}{l}\text { Existe conciencia de la realidad multicultural que acoge el centro por parte de todos } \\
\text { los miembros de la comunidad educativa. }\end{array}$ & & \\
\hline El ambiente del centro refleja la interculturalidad y cultiva el diálogo intercultural. & & \\
\hline Existe voluntad decidida por parte del Equipo Directivo de avanzar hacia la integración. & & \\
\hline Existen entrevistas con familias para facilitar la acogida del alumnado extranjero. & & \\
\hline $\begin{array}{l}\text { Se coordinan las actividades interculturales del centro con proyectos más globales } \\
\text { (carácter social, comunitario, colaborativo). }\end{array}$ & & \\
\hline $\begin{array}{l}\text { Se realizan Jornadas interculturales donde se muestren y expliciten la diversidad } \\
\text { cultural del centro escolar }\end{array}$ & & \\
\hline $\begin{array}{l}\text { Se adscriben los alumnos/as inmigrantes a los cursos teniendo en cuenta la edad } \\
\text { cronológica sin tener en cuenta otros criterios (escolarización previa, conocimientos). }\end{array}$ & & \\
\hline $\begin{array}{l}\text { El centro dispone de protocolos para conocer la disponibilidad horaria de tutores para } \\
\text { mantener contactos con familias. }\end{array}$ & & \\
\hline
\end{tabular}




\begin{tabular}{|l|l|l|}
\hline \multicolumn{1}{|c|}{ Preguntas para el análisis } & si & no \\
\hline $\begin{array}{l}\text { Existe en el centro un plan de trabajo específico con las familias de los alumnos de } \\
\text { incorporación tardía. }\end{array}$ & \\
\hline $\begin{array}{l}\text { Existe en el centro unos criterios básicos para la adscripción al aula, teniendo en } \\
\text { cuenta los planteamientos para la atención a la diversidad. }\end{array}$ & \\
\hline $\begin{array}{l}\text { El centro tiene un Aula de Acogida donde se trabajan de manera intensiva las habi- } \\
\text { lidades comunicativas }\end{array}$ & \\
\hline Existe en el centro educativo un cuidadoso análisis de su contexto. & \\
\hline $\begin{array}{l}\text { Dentro del Plan de Atención a la Diversidad se recoge un Plan de Acogida, materia- } \\
\text { les pedagógicos, criterios de evaluación, adaptaciones curriculares, priorización de } \\
\text { refuerzos, actuaciones tutoriales y orientación profesional. }\end{array}$ & \\
\hline $\begin{array}{l}\text { El Consejo Escolar ante la llegada de alumnado inmigrante ha revisado críticamente } \\
\text { el PEC y ha analizado la coherencia de la PGA y los principios educativos de dichos } \\
\text { documentos }\end{array}$ & \\
\hline $\begin{array}{l}\text { Se abordan de inmediato situaciones de rechazo o menosprecio que puedan darse en } \\
\text { la escuela, sea con el grupo clase o con otros alumnos, tratándolo con las personas } \\
\text { implicadas. }\end{array}$ & & \\
\hline Hay criterios específicos sobre la evaluación del alumnado extranjero. & \\
\hline
\end{tabular}

Fuente: García (2003, p. 38)

Dada la estrecha vinculación que existe entre este instrumento y nuestro estudio, consideramos oportuna su utilización. El cuestionario nos ha servido para realizar un análisis inicial de diversos documentos institucionales y para tener una visión acerca de cómo es abordada la atención a la diversidad cultural del alumnado en tales documentos de centro.

\subsection{Procedimiento y análisis de la información de las entrevistas en profundidad}

Hemos puesto especial esmero en que sean los participantes (profesorado, alumnado y familias) quienes nos aporten pistas acerca de dónde poner nuestra mirada. Así pues, hemos reconstruido las categorías a partir de la información obtenida en las entrevistas en profundidad. Para su realización técnica partimos del modelo de Pascual et al (2003). A modo de ejemplo presentamos la tabla 4 donde reflejamos categorías emergentes en las entrevistas realizadas al profesorado:

TABLA 4

\section{Categorías emergentes en las entrevistas al profesorado}

\begin{tabular}{|c|}
\hline Categorías emergentes \\
\hline Diversidad cultural \\
\hline Participación familias \\
\hline Convivencia entre culturas \\
\hline Aspectos culturales \\
\hline Acceso al currículo ordinario \\
\hline
\end{tabular}




\begin{tabular}{|c|}
\hline Tradición histórica \\
\hline Jornadas interculturales \\
\hline Integración alumnado extranjero \\
\hline Aspectos lingüísticos \\
\hline Aspectos organizativos \\
\hline Aspectos curriculares \\
\hline Freire y la educación intercultural \\
\hline
\end{tabular}

El análisis de las entrevistas lo efectuamos a partir de categorías emergentes en el proceso de la entrevista. Una vez organizada la información de las diferentes entrevistas, realizamos un análisis cualitativo de las mismas a partir de la información obtenida por cada grupo de informantes.

\subsection{Procedimiento y análisis de la información de la observación participante}

Sin duda, las notas de campo, el diario de investigación y las fotografías realizadas suponen un aporte interesante que acompaña a la descripción del ambiente y del contexto en el que nos encontramos. Siguiendo a Patton (citado en Colás, 1998, p. 272) la observación en el campo la hemos centrado en los siguientes aspectos que presentamos en la figura 3 :

\section{FIGURA 3}

\section{Aspectos para realizar la observación participante}

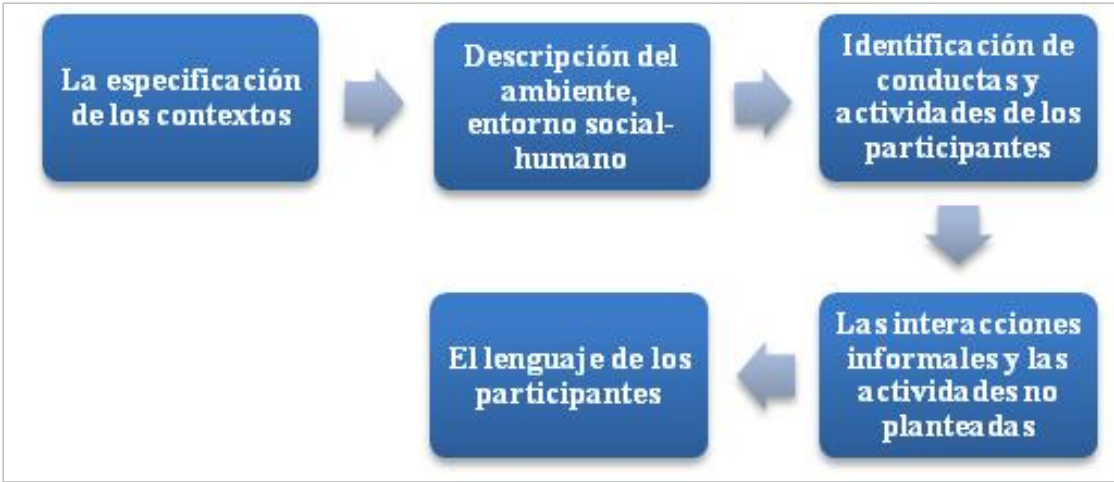

Una vez recogida la información hemos realizado un análisis cualitativo de la misma poniendo nuestra mirada en tales aspectos estableciendo conexiones con los objetivos de nuestro estudio. 


\subsection{Procedimiento y análisis de la información del grupo de discusión con el alumnado de Educación Secundaria Obligatoria}

La utilización de esta técnica de recogida de información, está justificada porque nos brinda la posibilidad de conversar libremente con el alumnado y hacernos eco de sus opiniones, percepciones, creencias, etc. El hecho de realizar diversos grupos de discusión -cinco grupos-, nos brindó una visión holística de la opinión del alumnado. A pesar de que partíamos de categorías de análisis previas, durante el grupo de discusión emergieron temas de especial interés para el alumnado que también analizamos, como así representamos en la figura 4 :

\section{FIGURA 4}

Categorías de análisis utilizadas en los grupos de discusión

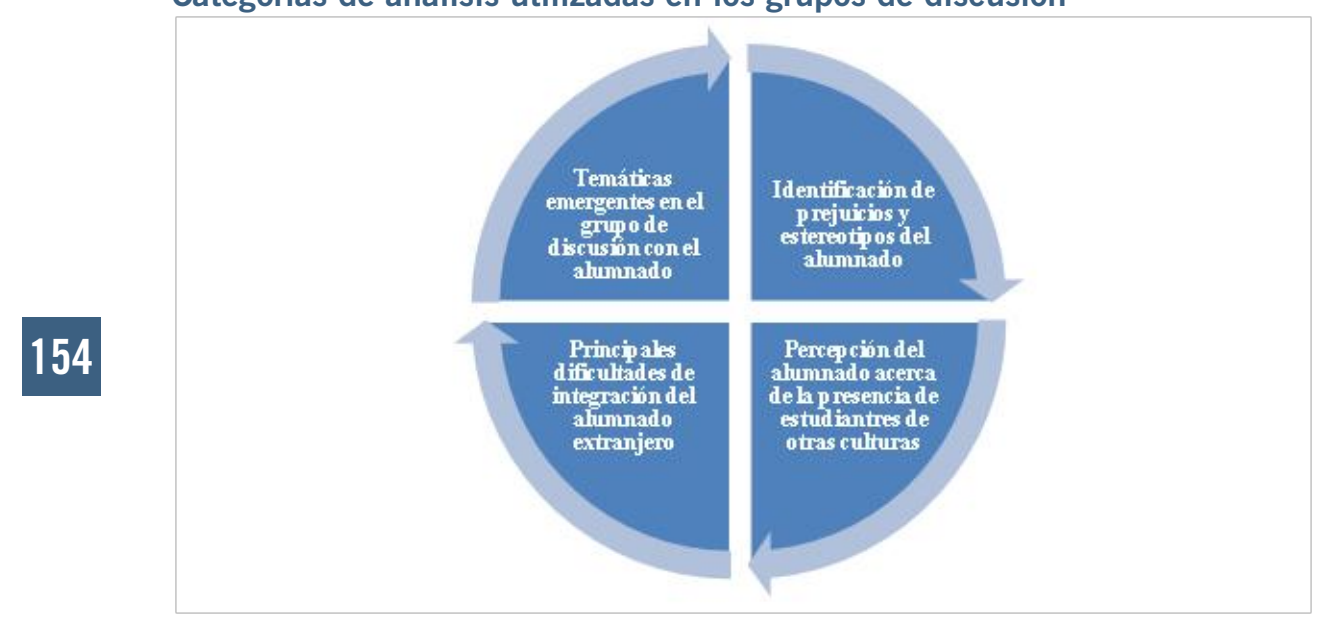

Una vez recogida la información en el grupo de discusión con el alumnado, realizamos el análisis cualitativo de la misma teniendo en cuenta tanto categorías previas como categorías emergentes en el proceso del grupo de discusión. 


\section{RESULTADOS}

\subsection{Principales resultados del análisis documental: bibliografía de Paulo Freire}

La utilización de la técnica del análisis documental y de contenido ha sido útil en el contexto de nuestra investigación, ya que nos ha permitido realizar un estudio crítico y en profundidad de la obra de Freire e interpretar, con la visión actual, ideas centrales de su pensamiento. A modo de síntesis, ofrecemos un resumen en los siguientes apartados:

- Escuela permanentemente abierta al cambio. Para Freire enseñar exige el reconocimiento y la asunción de la identidad cultural del educando.

- Reflexión sobre la práctica. Freire considera que los saberes necesarios para la práctica educativo-crítica con base en una ética-pedagógica se basan, entre otros aspectos, en la investigación, la actitud crítica, la humildad, el buen juicio, la tolerancia o la curiosidad.

- Justicia social. Nuestro autor, en efecto, es una persona que tuvo una especial preocupación por el otro, por denunciar las injusticias y por mostrarnos que los seres humanos tenemos en nuestras manos las posibilidades de transformación.

- Voluntad política de cambio. Freire alude sistemáticamente a la imposibilidad de considerar la práctica educativa como neutra o como una actividad que pueda ser abordada exclusivamente desde una dimensión técnica o metodológica.

- Diversidad lingüística. Para Freire la educación intercultural debe estar abierta a reconocer la diversidad cultural y lingüística. Ello implica el mantenimiento y aprendizaje en la lengua materna, así como la opción de contar con docentes de culturas y lenguas minoritarias en las aulas y escuelas.

- Educación problematizadora frente a educación bancaria. Una de las principales aportaciones de Freire a la educación intercultural se centra en el análisis crítico de las problemáticas socioculturales, frente a la concepción bancaria, es decir, la centrada en la mera trasmisión de contenidos académico-culturales.

- Materiales curriculares al servicio de la comprensión del contexto. Frente a la preocupación por la memorización mecánica, propia de los libros de texto, Freire propugna la elaboración de materiales curriculares que despierten la curiosidad de los 
alumnos para interpretar dialécticamente los contextos en los que interactúan.

\subsection{Principales resultados del análisis documental: materias del currículo asturiano}

- La mayoría de las materias del currículo asturiano contemplan en los elementos analizados -objetivos, contenidos, orientaciones metodológicas, criterios de evaluación, contribución a las competencias básicas- aspectos inequívocamente relacionados con lo que entendemos por educación intercultural.

- En la formulación de los objetivos generales y contenidos, varias materias contemplan aspectos relacionados con la valoración de la diversidad cultural, la tolerancia, la consideración de otras culturas y la preservación del patrimonio artístico y cultural. Sin embargo es preciso destacar que tal diversidad queda "restringida" al ámbito autonómico, español y europeo, obviando otras realidades presentes en las aulas.

- Una de las dimensiones de análisis en las que está más presente una perspectiva intercultural es la relacionada con las orientaciones metodológicas, dado que se apuesta una metodología basada en el diálogo como instrumento facilitador del aprendizaje, el empleo de una metodología participativa y colaborativa, donde el docente desempeña un papel facilitador del proceso de enseñanza-aprendizaje.

- Con relación a las competencias básicas se evidencia claramente que dicho documento enfatiza la importancia de la perspectiva intercultural. Así, se subraya la importancia de cultivar la sensibilidad, la creatividad y el pensamiento divergente; promover la tolerancia, apreciar los rasgos de identidad así como las diferencias, habilidades y destrezas relacionadas con la convivencia cívica.

- Con relación al apartado de los criterios de evaluación, destacamos que en la mayoría de las materias se formulan criterios de evaluación relacionados con aspectos tales como investigar, reflexionar, cooperar, valorar, etc. Sin embargo, se echa en falta el establecimiento de unos procedimientos claros y operativos para efectuar tal evaluación. 


\subsection{Principales resultados del análisis documental: documentos institucionales}

La problemática de la atención a la diversidad cultural del alumnado no es ajena a los planteamientos que rigen la organización de la vida en el centro. Tal problemática se puede sintetizar en los siguientes aspectos:

- A pesar de que el IES alberga un elevado número de alumnado extranjero, en el Proyecto Educativo del Centro no existen objetivos concretos relacionados con la atención a tal diversidad cultural.

- Ante una realidad y contexto social cambiante en el IES, no se ha realizado ninguna revisión ni evaluación de las actitudes del profesorado respecto a la diversidad cultural que existe en el centro.

- Existe un Plan de Acogida concreto que contempla diversas actuaciones al inicio de curso o bien cuando se incorpora un nuevo alumno o alumna y, en ese sentido, es muy favorable la existencia de un protocolo de actuación que establece los pasos a seguir en lo que respecta a la acogida al alumnado extranjero.

- El IES cuenta con Aulas de Acogida y de Inmersión Lingüística, situación que contribuye favorablemente al desarrollo de competencias comunicativas y también al desarrollo de habilidades socioeducativas del alumnado.

\subsection{Principales resultados de las entrevistas a profesorado (experto)}

- Los informantes perciben la presencia de alumnado extranjero como un fenómeno social de gran relevancia en la vida del IES y como una situación que tendrá continuidad en el tiempo.

- En general, perciben dicho fenómeno como un reto educativo y como una circunstancia que necesariamente conlleva cambios pedagógicos y metodológicos.

- A pesar de percibir la presencia de alumnado extranjero en el centro como una oportunidad, también existe la percepción de que hay problemas derivados, justamente, de las circunstancias en que la inmigración accede a la escuela.

- Algunas dificultades más notables que tiene la escolarización del alumnado extranjero son el gran desfase curricular, diversos ritmos de aprendizaje, desconocimiento de la lengua de aprendizaje, etc. 
- Coinciden en señalar que las peculiaridades culturales del alumnado extranjero representan un choque cultural importante para la comunidad educativa autóctona.

\subsection{Principales resultados de las entrevistas a otro profesorado}

- El progresivo aumento del alumnado extranjero en las aulas representa una de las principales preocupaciones del profesorado, sobre todo en lo relativo al desconocimiento de la lengua española.

- El profesorado destaca también que otra de las principales problemáticas que trae consigo este alumnado son las experiencias educativas previas, incluyendo aquí el hecho de que en ocasiones hay estudiantes que ni siquiera han sido escolarizados.

- El profesorado entiende que la escuela, y concretamente la escuela pública por su propia esencia, ha de ser la responsable de educar al alumnado extranjero en las mismas condiciones y con las mismas oportunidades que al alumnado autóctono.

- El profesorado también señala que, en general, no disponen de unos criterios pedagógicos claros para atender a la diversidad cultural existente en las aulas, de modo que cada docente actúa intuitivamente $y / 0$ de modo voluntarista.

\subsection{Principales resultados de las entrevistas a las familias autóctonas}

- La mayoría de familias valoran de forma positiva la diversidad cultural existente en el centro, aunque también hay algunas que consideran que tal diversidad puede condicionar el rendimiento escolar de sus hijos e hijas.

- Son conscientes de que existen determinados criterios organizativos en el centro -en torno al Programa Bilingüe- que favorecen que exista concentración del alumnado extranjero en determinados grupos marginales.

- Coinciden en señalar que la convivencia entre el alumnado es buena y reconocen que sus hijos tienen amigos pertenecientes a otras culturas.

- Valoran de positivamente que desde el centro se promuevan iniciativas relacionadas con el conocimiento entre culturas.

- En general, están abiertas a participar en este tipo de propuestas. Si bien es cierto que algunas manifiestan tener dificultades horarias u otros impedimentos que dificultan su participación en estas iniciativas. 


\subsection{Principales resultados de las entrevistas a familias extranjeras}

- Con relación al proceso de integración del alumnado no autóctono las familias extranjeras opinan que éste depende de factores variados. Así, consideran muy importante la edad temprana del alumnado para lograr el éxito de tal integración, así como el conocimiento previo de la lengua.

- La convivencia del alumnado extranjero con el alumnado asturiano no presenta, en principio, dificultades. Los problemas de convivencia se relacionan con otras problemáticas, comunes en todos los centros.

- Sí se percibe que hay aspectos del "choque cultural" que, en un momento dado y debido al desconocimiento de determinadas pautas, podrían ocasionar conflictos.

- Se muestran interesadas en que desde el centro educativo se desarrollen iniciativas encaminadas al conocimiento entre las culturas y al intercambio cultural.

- Estarían dispuestas a participar en iniciativas relacionadas con el conocimiento entre culturas, siempre y cuando sus obligaciones laborales no se lo impidieran.

\subsection{Principales resultados de la observación participante}

- El centro educativo elegido es muy rico por lo que se refiere a la calidad de sus recursos humanos. El profesorado tiene una media alrededor de diez años de experiencia en ese centro educativo.

- La mayoría tiene más años de experiencia en otros centros. En la plantilla hay docentes pertenecientes al cuerpo de catedráticos de secundaria y también hay doctores.

- EI IES está muy bien dotado de recursos materiales. Cuenta con unas buenas instalaciones y espacios pensados para que el alumnado esté en un entorno favorable para el estudio y el aprendizaje: aulas de tecnologías, laboratorios, materiales audiovisuales, aulas de dibujo, música, idiomas, etc.

- Es perceptible la relación entre espacios y modelos de enseñanza. No cabe duda de que nos encontramos en un IES en el que se puede apreciar una relación clara y directa entre espacios, contenidos y tratamiento metodológico de los mismos. 


\subsection{Principales resultados del grupo de discusión con alumnado de ESO}

- El alumnado autóctono percibe la presencia de alumnado extranjero en las aulas asturianas como un hecho natural que forma parte de la vida del centro educativo.

- La convivencia de alumnado de diversas culturas no representa, en principio, problema alguno y, por lo general, se dan buenas relaciones de amistad.

- Uno de los principales problemas del alumnado extranjero está relacionado con el aprendizaje de la lengua castellana y su utilización en el contexto académico. Dicho problema existe, incluso, para el alumnado latinoamericano que también manifiesta dificultades de comprensión y expresión oral y escrita en actividades académicas de aula.

- Existe un interés -alumnado extranjero y autóctono-, porque se produzcan procesos de intercambio cultural.

- El alumnado, extranjero y autóctono, manifiesta tener poco conocimiento de las culturas presentes en las aulas y a las cuales pertenecen sus propios compañeros y, al mismo tiempo, demuestran por sus comentarios que sus percepciones previas acerca de otras culturas están contaminadas, de estereotipos y prejuicios.

- El alumnado extranjero valora positivamente la cultura asturiana y a pesar de que refiere que el cambio de una cultura a otra es un proceso difícil, en general, se sienten "a gusto". A ello contribuye notablemente el aprendizaje de la lengua y las relaciones de amistad de su grupo de iguales.

- Con relación a la lengua asturiana, se aprecia la pervivencia de la diglosia, especialmente visible en el alumnado autóctono, ya que en ocasiones no valoran el valor cultural de la lengua autóctona. La actitud de los alumnos extranjeros es, en este sentido, de extrañeza, curiosidad e interés.

\section{CONCLUSIONES}

Para exponer las conclusiones del estudio, lo haremos en torno a las 5 cuestiones clave sobre las que se ha centrado nuestra investigación. 


\subsection{Aportaciones de Paulo Freire a la educación intercultural.}

Nuestro estudio evidencia que la problemática de la educación intercultural no es ajena a los grandes intereses educativos del gran pensador brasileño, sino que, al contrario, está muy presente en toda su obra. Paulo Freire, en este sentido, puede y debe ser reivindicado como un precursor en la defensa de los valores educativos del diálogo intercultural. Así, por un lado, la escuela popular y liberadora en la concepción freireana, debe estar atenta para asumir la identidad cultural del educando y debe reconocer los valores de la diversidad cultural y lingüística, y, por otro, debe ser capaz de analizar críticamente las problemáticas socio-culturales del contexto en que tal educación popular tiene lugar.

\subsection{Retos que la diversidad cultural plantea al currículo asturiano}

A continuación presentamos los principales retos que la diversidad cultural plantea al currículo asturiano estableciendo, en su caso, posibles conexiones con la pedagogía de Freire. Lo haremos en torno a los siguientes aspectos:

- Respeto a la identidad cultural del alumnado. La atención a la diversidad cultural del alumnado implica el respeto e interés por la identidad cultural del alumnado y, en el caso de que sea de otra cultura, implica también el conocimiento de la realidad social de su país de origen y la reflexión acerca de su acercamiento a la realidad de las aulas asturianas. Igualmente, implica, interés por su cultura. En este sentido, la aportación de Freire se centra en aceptar y respetar la diferencia como valor educativo básico sin el cual la escuela no se puede dar.

- Valoración de la diversidad cultural o la multiculturalidad. En un contexto -ya sea educativo o social- en el que conviven diferentes culturas resulta altamente enriquecedor el hecho de compartir y aprender aspectos culturales diferentes, siempre desde el respeto entre culturas y teniendo como referente el marco de los Derechos Humanos. Aquí, el punto de contacto con Freire radica en el hecho de que nuestro autor propugna un modelo de escuela democrática que promueve el desarrollo de contenidos curriculares partiendo de una concepción antropológica de la cultura, de modo que sea posible cuestionarse a quién pertenece la cultura que se imparte en las escuelas y criticar el modelo monocultural y etnocéntrico.

- La interculturalidad como aspiración política. Desde el punto de vista político la atención a la diversidad cultural del alum- 
nado es uno de los retos de las sociedades del siglo XXI. Las políticas educativas propugnadas por organismos nacionales e internacionales (Consejo Europa, UNESCO) hacen una apuesta firme por el desarrollo de actuaciones educativas encaminadas a la transmisión de competencias interculturales. Precisamente Freire insiste en la necesidad de construir educativamente la interculturalidad y considera que es una creación histórica que implica decisión, voluntad política, movilización, organización de cada grupo cultural con miras comunes y que además exige una nueva ética política fundada en el respeto a las diferencias.

- Relación entre diversidad cultural y diversidad lingüística. Si hay diversidad cultural, en el medio y en la escuela, eso significa que también hay diversidad lingüística. Pues bien, la diversidad lingüística también tiene que estar presente en la realidad educativa de las aulas, de modo especial por lo que hace referencia a las lenguas minoritarias del entorno. Freire afirma que no existe bilingüismo y mucho menos multilingüismo fuera de la interculturalidad. Para él el lenguaje no es solo un instrumento de comunicación, sino, además, una estructura de pensamiento del ser nacional. Es una cultura y con un valor social y educativo fundamental.

- Un enfoque democrático de educación intercultural. Es fundamental clarificar el enfoque de educación intercultural que se quiere adoptar y desarrollar un modelo coherente con tales planteamientos, ya que en numerosas ocasiones se habla de un modelo en el que se favorezca la inclusión pero, finalmente, lo que se lleva a la práctica es un modelo asimilacionista. Freire también se refiere a la necesidad de buscar la coherencia entre la teoría y la práctica o, lo que es lo mismo, la coherencia entre el modelo de educación intercultural que queremos y el que finalmente desarrollan los docentes.

- Cultura accesible para los estudiantes vs. memorización mecánica de contenidos. A pesar de que los principios que inspiran nuestro sistema educativo y el currículo asturiano son otros, todavía nos encontramos ante prácticas de enseñanza academicista centradas en el aprendizaje acrítico de contenidos en lugar de una enseñanza comprensiva basada en la adquisición de competencias y aprendizajes básicos para vivir en sociedad y en la que se fomenta la curiosidad profunda del alumnado. A este respecto, Freire siempre criticó el excesivo uso de ejercicios repetidos que sobrepasan el límite razonable en cuanto dejan de lado una educación crítica de la curiosidad y afirma que 
muchas veces los docentes nos empeñamos en dar respuestas a preguntas que no nos fueron hechas.

\subsection{Ejes para la formulación de una alternativa inicial de educación intercultural}

Como se recordará, uno de los objetivos de nuestra investigación consistía en elaborar una alternativa inicial de educación intercultural para la ESO de acuerdo con las aportaciones de Freire. Pues bien, tal alternativa podría sintetizarse en los siguientes puntos:

- La diversidad cultural ha de ser presentada a los estudiantes como un valor que enriquece la cultura propia y la de los demás. Ninguna cultura tiene que entenderse como superior a las demás, aunque sea la hegemónica o dominante.

- Los materiales han de estar libres del etnocentrismo -a veces, eurocentrismo- que impide apreciar los aspectos positivos de otras culturas o que imposibilita ver que el logro de una sociedad más equilibrada no constituye ninguna utopía.

- Una propuesta intercultural coherente debe ofrecer la posibilidad de valorar críticamente la cultura propia y la del otro para ser conscientes de que no todas las tradiciones culturales pueden ser valoradas y asumibles actualmente.

- Se han de fomentar actividades interculturales constructivas que valoren la cultura de los diferentes pueblos en forma de sociedades modernas, capaces, inteligentes, dignas de libertad, con derecho al uso de su lengua propia, etc.

- La educación intercultural tiene que ser capaz de problematizar y analizar las causas que provocan los conflictos existentes, tanto dentro de nuestra sociedad como los que se producen en otros pueblos y culturas.

- El diálogo es la base de la educación intercultural y tiene que producirse con humildad, coherencia y rigor, respetando la opinión del otro. La concepción dialógica ha de contemplar el entendimiento mutuo entre personas y culturas.

- Las actividades tienen que tener como marco referencial los derechos consagrados internacionalmente (derechos humanos, del niño, lingüísticos, etc. ). Además, han de transmitir el rechazo de la violencia, la esclavitud, o la crueldad de la guerra. 
- La metodología debe partir de los saberes con los que los alumnos llegan al aula, pero poniendo de manifiesto que a veces tales saberes no son tales, sino basados en prejuicios y estereotipos que hay que superar

- En la educación intercultural no es exigible la neutralidad política, pero sí unos mínimos principios éticos que aseguren el no adoctrinamiento del alumnado y el respeto por ideas políticamente democráticas y divergentes.

\subsection{Posibilidades de aplicación a partir de un estudio de caso en un IES asturiano}

Las implicaciones didácticas y pedagógicas que de tal alternativa pudieran derivarse se refieren, específicamente, al contexto en el que hemos realizado nuestro estudio. No consideramos, por tanto, que sea un criterio riguroso hacer generalizaciones sin estudiar previamente las características del contexto de aplicación. Las conclusiones, en este sentido, hacen referencia a que:

- El IES considerado constituye un buen marco para la puesta en práctica de dicha alternativa inicial de actividades de educación intercultural, dado que nos encontramos en un centro en el que conviven diversidad de culturas.

- Existe una necesidad latente y sentida, tanto entre el profesorado como entre el alumnado y las familias, por el desarrollo de iniciativas relacionadas con la educación intercultural y, según las entrevistas realizadas, la comunidad educativa, estaría dispuesta a participar en la elaboración de propuestas al efecto.

- La propuesta podría constituir un interesante recurso para el profesorado, dado que representaría un apoyo al trabajo docente. En ese sentido, el profesorado, reclama que se le brinden materiales para atender a la diversidad cultural.

- Dicha alterativa inicial está planteada desde una perspectiva integral y contempla de forma explícita la adquisición de competencias básicas propuestas en la etapa de la ESO y supone una forma de motivar y concienciar al alumnado.

Sin embargo, es necesario ser humildes -y también valientes- para reconocer que, pese a esta realidad, la posibilidad de desarrollar tal alternativa de educación intercultural se requiere que, previamente, se den una serie de condiciones que a continuación comentamos y sin las cuales dicha alternativa no sería viable o, simplemente, representaría una solución parcial en un 
contexto educativo en el que convergen fenómenos ciertamente complejos. Como indica San Fabián (2011) la intervención educativa no puede obviar lo organizativo. Por ello, comentamos algunos requisitos que, necesariamente, han de darse si queremos llevar a término dicha propuesta. Tienen que ver con los siguientes aspectos:

- La existencia de una infraestructura organizativa en el IES en la que la vida diaria (académica y relacional) se "interculturalice".

- Entender el sentido educativo y social de la enseñanza secundaria y desarrollar un modelo de enseñanza comprensiva que tenga en cuenta los diferentes ritmos de aprendizaje, expectativas, motivación y necesidades de un alumnado diverso.

- Apostar por un modelo de currículo en el que tenga cabida la diversidad cultural del alumnado y que su desarrollo en las aulas implique ayudar al alumnado a construir su propia visión del mundo.

- Todo ello tiene que producirse en un contexto en el que el alumnado tenga las mismas oportunidades educativas. La Educación no puede convertirse en el privilegio exclusivo de quienes puedan pagarla.

\subsection{Principios, básicos y coherentes, de educación intercultural}

De acuerdo con los resultados y conclusiones anteriores, se pueden establecer con carácter general, unos principios básicos de educación intercultural. Así:

- Un modelo de escuela intercultural es una escuela permanentemente abierta al cambio y a la mejora. Ha de ser un modelo de escuela flexible, democrática, inclusiva y que, como señala Freire, reconoce la diferencia como un valor.

- Una escuela intercultural requiere operar en términos de humanidad y superar los etnocentrismos más primarios. Es necesario cuestionar aspectos asociales de nuestra cultura y fomentar el respeto por otras personas y su cultura.

- Es necesario re-plantear el modelo de formación y organización del profesorado, puesto que una escuela multicultural, necesariamente ha de contar con profesorado sensible ante otras culturas y que pueda enseñar y valorar la lengua materna de los estudiantes. 
- La educación intercultural, de acuerdo con Freire, implica un proyecto pedagógico claramente orientado a favorecer el cambio social, entendido éste en el sentido de buscar principios de justicia social y una ética fundada en el derecho a las diferencias.

- Este modelo de educación intercultural, necesariamente, ha de contemplar un sistema de evaluación crítica y orientada a la mejora de la propia práctica docente. Para ello se pueden utilizar instrumentos y procedimientos de evaluación alternativos a los actuales.

- La educación intercultural implica la necesidad de reflexionar acerca de las injusticias y el sufrimiento (inmigración, pobreza, violencia contra las mujeres, etc.). Esto es, en esencia, lo que Freire entiende como "educación problematizadora".

- Una escuela intercultural ha de estar abierta a la comunidad educativa y debe favorecer mecanismos de participación del profesorado, alumnado, familias y colectivos sociales. Es un modelo de escuela democrática que además de estar abierta permanentemente a la realidad contextual de los educandos como soñaba Freire- también debe estar dispuesta a aprender de sus relaciones con el medio.

- La educación intercultural conlleva necesariamente trabajar con diversidad de fuentes y recursos. Implica que el alumnado sea protagonista del proceso de enseñanza-aprendizaje y se convierta en sujeto activo de dicho proceso, utilizando recursos que despierten la motivación y el interés por aprender.

- Una escuela multicultural conlleva revisar la selección cultural de los contenidos que conforman el currículo común para hacerlo más universal y representativo de las culturas y de la realidad social. En la selección de los contenidos curriculares -como recuerda Freire- es necesario preguntarse por cuestiones tales como: ¿a quién pertenece esa cultura? o ¿a favor de quién está esa cultura? Cuestiones que nos servirán para comprender que, en definitiva, ninguna práctica educativa es neutra.

- El diálogo es una exigencia existencial, por ello Freire lo propone como método de conocimiento. También señala que tiene que producirse con respeto y atendiendo a los argumentos del otro. Una escuela multicultural debe favorecer que se produzcan procesos de comunicación dialógica que puedan modificar, en su caso, ideas erróneas o preconcebidas. 
Nuestro estudio, en definitiva, no deja de ser un intento más de contribución para "repensar" y "reinventar" las ideas de Paulo Freire y adaptarlas a la necesidad social y educativa de plasmar una educación intercultural cada vez más necesaria en el complejo y diverso mundo actual. Si modestamente hemos ayudado a tal pretensión nos daríamos por plenamente satisfechos.

\section{BIBLIOGRAFÍA}

Aguado, Ma.T. (2011). El enfoque intercultural en la búsqueda de buenas prácticas escolares. Revista Latinoamericana de Inclusión Educativa, 5(2), 23-42.

Banks, J. A. (2015). Cultural diversity and education. Routledge.

Besalú, X. (2002). Diversidad cultural y educación. Madrid: Síntesis.

Colás, P. (1998). Análisis cualitativo de datos. En Buendía; L.; Colás, P. y Hernández Pina, F. Métodos de investigación en psicopedagogía. (pp. 288-310). Madrid: McGraw-Hill

Díez Gutiérrez, E.J. (2014). La práctica educativa intercultural en Secundaria. Revista de Educación, 363, 12-34.

Durkheim, E. (1975). Educación y Sociología. Barcelona: Ediciones Península.

Freire, P. (1969). Educación como práctica de la libertad. Madrid: Siglo XXI.

Freire, P. (1970). Pedagogía del oprimido. Madrid: Siglo XXI.

Freire, P. (1993). Pedagogía de la Esperanza: un reencuentro con la pedagogía del oprimido. Madrid: Siglo XXI.

Freire, P. (1994). La naturaleza política de la educación. Barcelona: Planeta De Agostini.

Freire, P. (1997). Pedagogía de la Autonomía. Saberes necesarios para la práctica educativa. Madrid: Siglo XXI.

Freire, P. (1997a). A la sombra de este árbol. Barcelona: El Roure Editorial

Freire, P. (2003). El grito manso. Madrid: Siglo XXI.

Freire, P. (2006). Pedagogía de la tolerancia. México: Centro de Cooperación Regional para la Educación de Adultos en América Latina y en el Caribe. CREFAL.

Freire, P. (2010). Pedagogía de la indignación. Madrid: Ediciones Morata S.L.

Freire, P. (2012). Cartas a quien pretende enseñar. Madrid: Biblioteca Nueva S.L.

García, J.M. (2003). Cuestionario para la evaluación del tratamiento de los procesos de interculturalidad en los centros educativos. En F. Luengo y H. Ramos (Coord.). Interculturalidad y Educación: Un nuevo reto para la sociedad democrática (pp. 37-44). Proyecto Atlántida.

García Castaño, F.J.; Pulido, R.A. y Montes, A. (1997). La educación multicultural y el concepto de cultura. Revista Iberoamericana de Educación, 13, 223-256. Recuperado de: https://rieoei.org/RIE/article/view/1143 
Gimeno, J. (2005). La Educación Secundaria Obligatoria: Su sentido educativo y Social. Madrid: Morata.

Goetz, J.P. y LeCompte, M.D. (1988). Etnografía y diseño cualitativo en investigación educativa. Madrid: Morata.

González Riaño, X.A. (1994). Interferencia lingüística y escuela asturiana. Oviedo: Academia de la Llingua Asturiana.

Lynch, J. (1991). Education for citizenship in a multicultural society. Cassell.

Louzao, M. (2009). Diseño, desarrollo y evaluación de un proyecto de educación intercultural en un centro asturiano de Educación Primaria. Una perspectiva de la investigación en la acción y el estudio de caso. [Tesis Doctoral]. Universidad de Oviedo.

Louzao, M. y González Riaño, X.A. (2007). La integración social y educativa del alumnado inmigrante en Asturias. Un estudio de Caso. En: Los premios Nacionales de Investigación educativa y Tesis Doctorales 2005, (pp. 11-41). Madrid: CIDE.

Merino, J. y Muñoz Sedano, A. (1995). Eje de debate y propuestas de acción para una pedagogía intercultural. Revista de Educación, 307, 127-162. (Monográfico Educación Intercultural).

Pascual, J.; Mori, M.; González Riaño, X.A. y Atienza, J.L. (2003). Interculturalidad y diversidad lingüística: Estudio de las actitudes lingüísticas en Asturias y sus implicaciones didácticas. Oviedo: Ediciones KRK.

Pérez Gómez, A.I. (1994). Las funciones sociales de la escuela: de la reproducción a la construcción crítica del conocimiento y la experiencia. En Gimeno, J. y Pérez Gómez, A.I. Comprender y transformar la enseñanza, (pp.17-33). Madrid: Morata S.L

Pérez Tapias. J.A. (2010). Educar desde la interculturalidad. Exigencias curriculares para el diálogo entre culturas. En Gimeno, J. (Comp.). Saberes e incertidumbres sobre el Currículum, (pp. 149-161). Madrid: Morata S.L.

Sáez Alonso, R. (2006). La Educación Intercultural. Revista de Educación, 339, 859-881.

San Fabián, J.L. (2011). El papel de la organización escolar en el cambio educativo: la inercia de lo establecido. Revista de Educación, 356, 41-60.

Sleeter, C.E. y Grant, C.A. (1999). Making choices for multicultural education: Five approaches to race, class, and gender. New York, NY: Wiley.

Torres Santomé, X. (2008). Diversidad cultural y contenidos escolares. Revista de Educación, $345,83-110$.

Verdeja, M. (2015). Aportaciones de la pedagogía de Paulo Freire a la educación intercultural. Posibilidades de aplicación al sistema educativo en Asturias. [Tesis Doctoral]. Universidad de Oviedo. 